\title{
Automated Construction of Variable Density Navigable Networks in a 3D Indoor Environment for Emergency Response
}

\author{
Submitted: $15^{\text {th }}$ July 2015
}

Revised: $1^{\text {st }}$ December 2015, 20 $0^{\text {th }}$ April 2016 and $10^{\text {th }}$ August 2016

Pawel Boguslawski ${ }^{1,}{ }^{*}$, Lamine Mahdjoubi $^{1}$, Vadim Zverovich $^{1}$, Fodil Fadli ${ }^{2}$

${ }^{1}$ Faculty of Environment and Technology, University of the West of England, Bristol, United Kingdom

2 Department of Architecture and Urban Planning, College of Engineering, Qatar University, Doha, Qatar

* Corresponding author, e-mail: pawel.boguslawski@uwe.ac.uk

Contact details:

Pawel Boguslawski

Department of Architecture and the Built Environment

Faculty of Environment and Technology

University of the West of England

Frenchay Campus, Coldharbour Lane

Bristol, BS16 1QY, United Kingdom

e-mail: Pawel.Boguslawski@uwe.ac.uk

e-mail: pboguslawski@gmail.com

tel.: +44 1173287019

mobile: +447956044921 


\section{Automated Construction of Variable Density Navigable Networks in a 3D 2 Indoor Environment for Emergency Response}

Pawel Boguslawski ${ }^{1,}{ }^{*}$, Lamine Mahdjoubi $^{1}$, Vadim Zverovich ${ }^{1}$, Fodil Fadli $^{2}$

${ }^{1}$ Faculty of Environment and Technology, University of the West of England, Bristol, United Kingdom

${ }^{2}$ Department of Architecture and Urban Planning, College of Engineering, Qatar University, Doha, Qatar

* Corresponding author, e-mail: pawel.boguslawski@uwe.ac.uk

\section{Abstract}

Widespread human-induced or natural threats on buildings and their users have made preparedness and rapid response crucial issues for saving human lives. The ability to identify the paths of egress during an emergency is critical for rescue and emergency services. Quality models supporting real, or near-real, time decision making and allowing the implementation of automated methods are very important. In this paper, we propose a novel automated construction of the Variable Density Network (VDN) for determining egress paths in anemencydangerous environments. VND is used for deriving a navigable network in an indoor building environment, including a full 3D topological model. The accuracy of the proposed paths prediction tool was compared with key methods for navigable network generation, using the actual floor plan of Doha World Trade Centre. Findings revealed that in comparison to prevailing approaches, a key benefit from this approach is an increased prediction accuracy of egress route planning.

Keywords: navigable networks, indoor navigation, emergency response, 3D modelling, topological models

\section{Introduction}

Destructive disasters caused by events such as fire, storm, or explosion result in damages and structural instability of buildings. Such disasters are rare but inevitable, and in the urban environment with high-rise and big public buildings they lead to casualties and occupants trapped inside. In this type of crisis, the challenge is to assist the search and rescue personnel in quickly preparing a plan to locate and rescue the surviving victims. Disasters such as the fire at Villagio shopping mall in Doha in May 2012, resulted in 19 people killed including 13 children, are reminders that effective preparedness and response might eliminate or reduce human errors and panic leading into the loss of lives (Scharfenort, 2012).

Tashakkori et al. (2015) emphasized the importance of situation awareness available for first responders in emergency response systems. The knowledge about indoor structure, occupancy or localization of fire utilities is minimal prior their arrival to the emergency scene. Thus, assessment of the scene and wrong decisions increase the response time. Therefore, actual and constantly updated information about the indoor situation, close outdoor area and optimal routes to selected points inside a building is crucial. This information includes, to name a few: floor plans, material types, location of hazardous materials, occupancy information, space ownership, location of water resources, fire and emergency utilities, etc. 
In an emergency, up-to-date information is needed for coordination, communication and efficient decision making (DeCapua and Bhaduri, 2007). Availability of information about the indoor structure is critical for simulation of emergency-movement of people and egress way finding for individual pedestrians and groups of people (Nelson and MacLennan, 1995; Pauls, 1995). Usually the focus is put on evacuation paths, flow of people and time necessary to leave a building (Kuligowski, 2004). However, the opposite paths, from outdoor to trapped people at certain locations in a building are taken by search and rescue teams. In such a scenario, a quick location of first responders and occupants is of great importance in search and rescue operations (Li et al., 2014). In the case of emergency, standard path finding using normal navigation routes may not be sufficient, as they may be too risky or not available due to damage (Liu and Zlatanova, 2012). In these circumstances, the ability for emergency and rescue personnel to identify alternative and optimal paths becomes critical.

Zverovich et al. (2016) proposed a new method for calculating safe paths taking hazard proximity into consideration. Standard navigable networks are not sufficient to perform hazard analysis. They include only connections available for pedestrians, whereas topological relations between adjacent rooms without doors in-between are not reflected. This applies not only for adjacency in horizontal direction but also vertical, through slabs. However, these connections are important in simulation of several hazard types, such as fire spread or explosion impact. Thus, 3D properties included in a model, e.g. spatial relationship in all directions, are essential for emergency response applications which go beyond evacuation path-finding.

This paper reports on the development of a new automated method for deriving a navigable network in a 3D environment, including full 3D topological model, which may be used for finding alternative egress routes and simulating phenomena associated with emergency responsesituation. The motivation of this research is to provide better situation awareness to the first responders prior to their arrival to the emergency-scene.

\subsection{Navigable models}

Available navigable models in emergency response research focus on standard path finding using doors and corridors (Goetz and Zipf, 2011; Kwan and Lee, 2005; Liu and Zlatanova, 2012; Vanclooster et al., 2014; Yang and Worboys, 2015). Most of these approaches retrieve a navigable model from 2D floor plans, while the 3D properties of the original environment are not always reflected in the model; even when the original 3D model is available. Very often, adjacent floors are connected only by vertical connections representing staircases, and spatial relationships between adjacent rooms sharing a ceiling/floor or a wall without a door are not reflected in these models. The resulting networks are often simplified in order to reduce the storage cost and improve path-finding computation time, which makes them unsuitable for alternative path finding or simulation of phenomena related to the emergency.

Various researchers propose more sophisticated models (Lamarche and Donikian, 2004; Lee, 2004; Liu and Zlatanova, 2012; Stoffel et al., 2007; Wenjie and Schneider, 2010), where a complex network is generated for special spaces intensively used for pedestrian navigation such as corridors or complex open spaces. This improves the navigable network within a building, because the original logical network, which reflects connections among adjacent rooms, is not an appropriate representation of navigation routes. The proposed automated methods are based on door and 
97

concave corner locations and visibility (Liu and Zlatanova, 2012), Straight Medial Axis Transformation (Lee, 2004), Delaunay Triangulation spatial subdivision (Krūminaite and Zlatanova, 2014; Lamarche and Donikian, 2004), and convex sub-regions partitioning (Stoffel et al., 2007). These methods often require input models with preserved consistency between the geometry and topology. In the case of incomplete of inaccurate floor plans, a network model may be interactively generated (Luo et al., 2014), but this may not be efficient in the case of emergency situations, when models for decision support should be available in a real or near-real time.

Indoor navigation including emergency navigation models have to meet several requirements (Brown et al., 2013; Wang and Zlatanova, 2013). Indoor spaces and their geometry included in an original building model should be classified based on predefined types and represented in the navigable model. Sub-division of spaces is one of the required functionalities, which makes the model suitable for accurate routing computation. Semantic information defining function, use or occupancy should be also reflected and attached to the relevant spaces. Static and moving objects that are obstacles for navigation are another elements, which should be incorporated in the model. Finally, connectivity among spaces based on their type has to be derived. These requirements are the most fundamental and are usually supplemented by other, more specific rules.

A slightly different approach to navigable network generation is utilized in robotic motion planning (Wallgrün, 2005). It is based on the Voronoi Diagram (VD) (Aurenhammer, 1991), where vertices and edges of the VD are used respectively to represent nodes and links in the network. This method may be used in an unknown environment, where the network is dynamically and automatically updated while the indoor scene is explored. Hierarchical structures are used to store networks in different granularity: from the coarsest to more detailed networks reflecting geometric details of the indoor environment. Different scales are used depending on required precision of path-planning.

Higher level of granularity may be achieved through a network densification similar to mesh densification utilized in the finite element mesh generation and refinement (Du and Gunzburger, 2002; Ho-Le, 1988; Petersen and Martins, 1997). Usually, mesh elements, e.g. triangles, are divided into smaller elements based on their centroids and original edges until the required level of granularity is achieved. Different criteria may be considered: the area of a mesh element or edge length. The size of the element is determined in a process (often iterative) minimizing an error between the exact solution and its finite element approximation (Du and Gunzburger, 2002; Dyck et al., 1992). A denser mesh may be generated locally in the vicinity of selected points (Chedid and Najjar, 1996).

Another field where densification of mesh is applied is geo-information: Triangulated Irregular Network (TIN), which is used to represent a digital model of terrain generated from point clouds and obtained from laser scanning. TIN is often densified using the Progressive TIN Densification (PTD) method (Axelsson, 2000; Zhang and Lin, 2013). New points are interpolated into a mesh representing the terrain which triggers division of a selected mesh triangle into smaller parts. The selection of a new point and the right triangle is based on specialized filters. Point candidates that do not belong to the terrain (e.g. buildings, trees, etc.) are calculated from their distance to TIN, and an angle between a mesh facet and a line connecting the point with the closest vertex of the facet. 
122 Other models for indoor navigation systems are presented by Afyouni et al. (2012). The biggest

123 drawback of these methods, in the context of applications other than standard indoor navigation, is

124 that not all spatial relations among adjacent rooms are available in the model. Usually, 2D plans are

125 used to reconstruct a navigable network within an individual building level. A link between two

126 rooms is created only if they share a door. Otherwise, where adjacent rooms share a slab or wall

127 without doors, adjacency relationship is not reflected in a model. Navigable networks for

128 consecutive floors are connected at certain points by additional links representing staircases (Lee

129 and Kwan, 2005).

130 However, some studies sought to fully utilise the 3D properties available from model reconstruction

131 (Becker et al., 2009; Boguslawski et al., 2011). Their methods are based on the Poincaré duality: in

132 practice the geometry of a volume is represented in the primal space using the boundary

133 representation (B-Rep) (Stroud, 2006), while a volume object (e.g. a room) is represented as a dual

134 node. A dual representation of a facet (e.g. a wall) is a dual edge bounded by dual nodes

135 representing adjacent rooms - a link. Therefore, the dual structure is a graph of connections among

$1363 \mathrm{D}$ objects. Attributes assigned to links may determine the navigability of the connections. These

137 links with attributes form a sub-graph of the initial graph of connections and may be called a

138 network.

139 The dual half-edge (DHE) proposed by Boguslawski (2011) is one of few data structures, which are

140 able to simultaneously store both the primal and the dual graphs. The construction of a model is

141 realized using CAD-like operators - Euler operators - where only the primal structure is explicitly

142 constructed while the dual is automatically updated (Boguslawski and Gold, 2010). Figure 1 provides

143 an example of a building represented with the DHE. In the model, doors and windows are

144 represented as zero-volume objects with associated dual nodes, which are part of the graph;

145 windows, which are embedded in a wall, are connected to the wall boundary using 'bridge' edges in

146 order to fulfil B-Rep requirements and avoid disconnections in the graph. A characteristic property of

147 the DHE model is the external volume enclosing the indoor model, which represents outside space.

148 The indoor model can be combined with the terrain model or transportation network by the

149 external cell (Boguslawski and Gold, 2015). The external volume is also considered in automatic exit

150 detection: a door adjacent to internal and external volumes is marked as an exit. 
a)

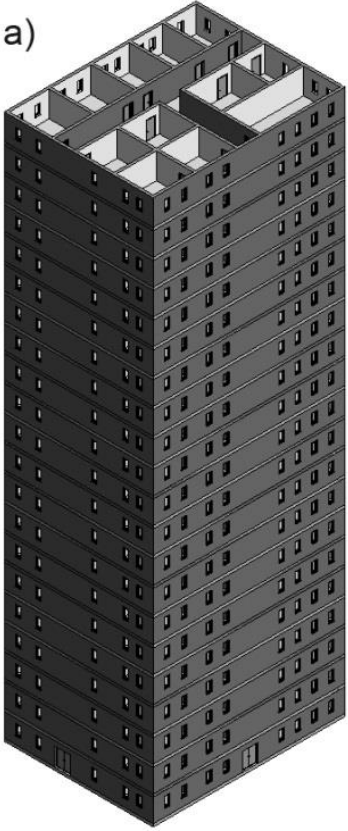

b)

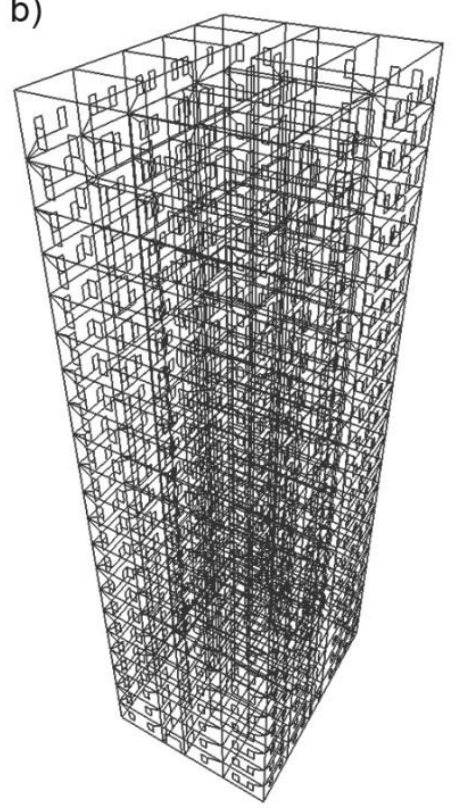

c)

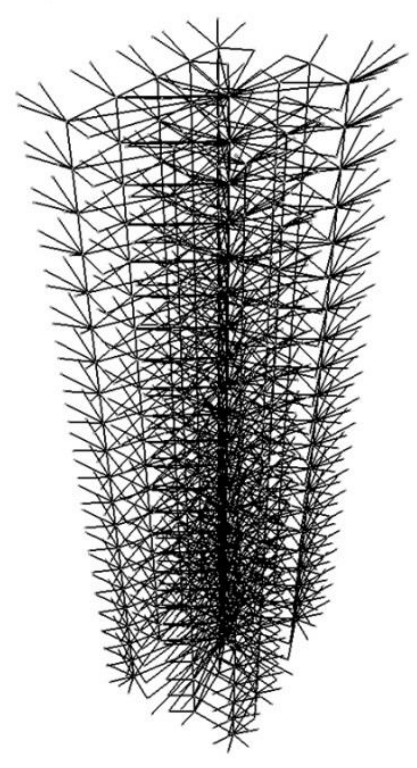

Figure 1. A 3D model of a building represented with the DHE data structure: a) an original BIM model; b) the DHE primal structure; c) the DHE dual graph of connections.

The dual graph, which reflects the adjacency relation between rooms, may be used to determine all the neighbours of a certain room. This graph is a network: rooms are represented by dual nodes; adjacent rooms are connected by a link; links can have different weights (i.e. attributes) associated with each direction. The network may be used for indoor navigation: paths between two locations can be calculated using graph algorithms (Boguslawski, 2011). A significant limitation of this representation is its applicability only to simple models with 'cubical' rooms. Complex rooms need to be manually partitioned into smaller sub-spaces with a specific configuration of doors in order to produce a network suitable for accurate indoor navigation. This issue will be addressed in the following section.

One of the advantages of the DHE representation is that not only links between adjacent rooms in the horizontal direction are present in the model, but all possible adjacency relations are reflected including those in the vertical direction. This is an important property, which is primarily used in hazard propagation simulation. The possibility of vertical navigation through staircases is automatically included in the model. Additionally, different weights for path-finding algorithms may be attached to links going upstairs and downstairs.

Regardless of a method used for navigable network generation, in order to share the resulting network and exchange between different applications, it should be represented in a common schema. The IndoorGML (OGC, 2014) is a new standard designed for indoor navigation applications, which complements other standards such as CityGML, KML and IFC. It consists of two main data models: topology of indoor environment and indoor navigation, which describe a network topology in built environment.

\subsection{Problem statement}

Figure 2a illustrates a close-up of a single level, the ground floor, in the building shown in Figure 1. The corridor (the grey area) is connected to adjacent rooms by doors and to the outdoors by exits A- 
C; there are three staircases S1-S3. The logical network representing connections among rooms at the ground floor is shown in Figure $2 \mathrm{~b}$. Other vertical connections between adjacent rooms at different levels are also included in the logical network (see Figure 1c). In a non-emergency scenario, only standard navigation routes via doors are used. These routes are represented by a sub-network of the logical network shown in Figure 2c.
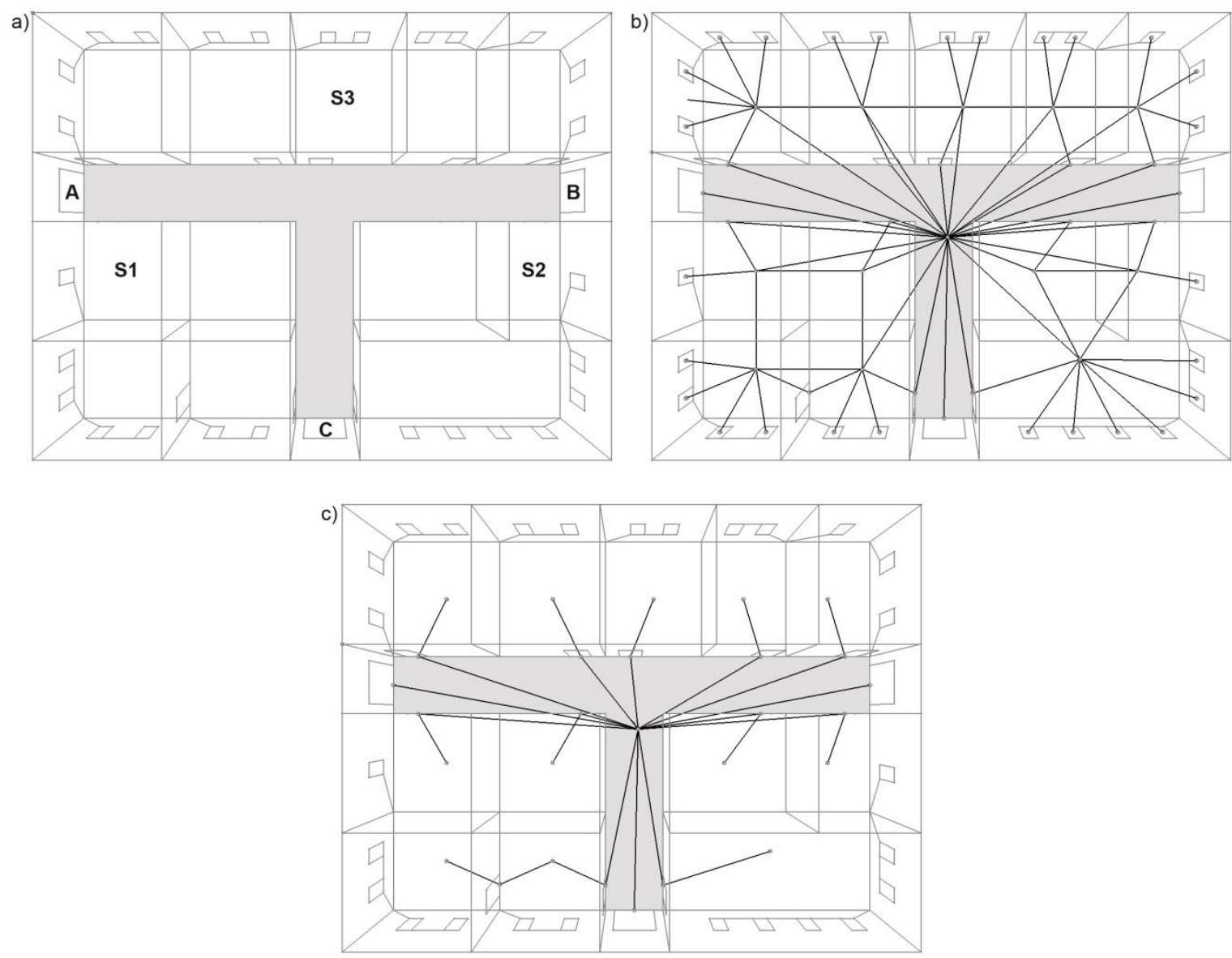

Figure 2. Ground floor of the building represented with the DHE: a) grey area represents a corridor with three exits A-C; S1-S3 are staircases; b) logical network; c) sub-network reflecting navigable connections through doors.

The network can be effectively used for navigation routes in simple models, e.g. when it consists of 'cubical' rooms with one door or two doors located on opposite walls. A problem arises when rooms have a complex shape and many doors (e.g. long corridors, concave rooms, big open spaces). If the shortest path from a room to the closest exit goes through a corridor node, located in the geometric centre, an incorrect exit may be selected (see Figure 3a). In reality, the shortest path between the graph nodes is calculated properly, but navigation routes within a corridor are not represented correctly. In addition, if the path finding algorithm is forced to select the correct closest exit ( $A$ in Figure $3 b$ ), then the path is not a 'natural' way of navigation - no one goes to the centre of a corridor first and then takes the correct door. A solution to the problem is to enhance the model by providing a better representation of 'natural' ways of indoor navigation. 

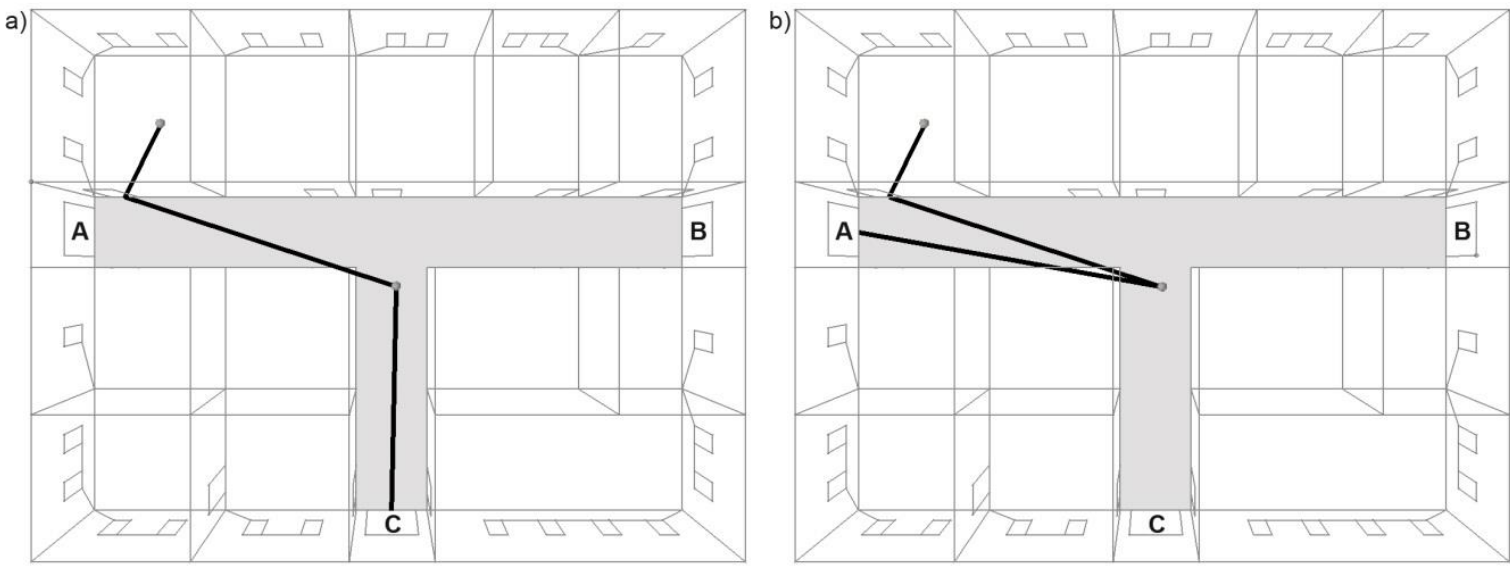

Figure 3. Paths (black lines) from a selected room through the corridor (grey area) to one of the exits ( $A-C$ are exits from a building): a) $C$ is wrongly calculated as the closest exit; $b$ ) $A$ is the closest exit but the path is not a 'natural' way of navigation.

The closest model is the one proposed by Liu and Zlatanova (2012), where the logical network representing a building structure is combined with navigable networks within single rooms: the network generation algorithm is based on the location of doors and concave corners. However, the navigable network is built from a spatial subdivision, the Voronoi tessellation, which is related to the method proposed by Lee (2004).

In this paper, a novel approach of navigable network generation, the Variable Density Network (VDN), based on the Voronoi Diagram is proposed. This approach allows a new automated method for deriving a navigable network in a 3D indoor environment, including a full 3D topological model, which may be used not only for standard navigation but also for finding alternative egress routes and simulating phenomena associated with disasters such as fire spread and heat transfer. The main application for the proposed network is calculation of egress paths in a dangerous environment to assist rescue teams, thus, evacuation of people and related topics, such as determination of position, route tracking, crowd simulation, human and social behaviour, are not taken into consideration. This can be a part of a bigger emergency response system.

The main focus of this research is a decision support system to assist emergency teams to determine egress paths in the event of emergency, thus, evacuation of people and related topics, such as crowd simulation, human and social behaviour, are not taken into consideration.

\subsection{Methodology}

In this research, two mock data models and a real-life case study were created in order to illustrate the proposed new method of navigable network generation (VDN). The first model is a high-rise office building with cubical rooms connected to a centrally located corridor (see Figure 4a). It is used to illustrate how the method works in narrow corridors of a complex shape with several doors to adjacent rooms. The second model is a two-storey building with a central big open space (see Figure 4b). It is used to show applicability of the same method to open spaces. The third model represents a floor plan of the Doha World Trade Centre in Qatar. This model is selected to carry out a comparative study between the proposed VDN and other prevailing models. The models were built in the Autodesk Revit design environment and exported to the gbXML data format. A gbXML file was imported and analysed in in-house software developed for testing purposes. Models are 
represented using the DHE data structure, which is an integral part of the in-house system. Some models may require validation in order to preserve a consistency between geometry and topology, which in particular concerns detection and fixing two problems: missing faces and overlapping between adjacent rooms. The workflow is shown in Figure 5.

a)

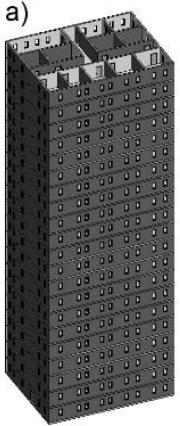

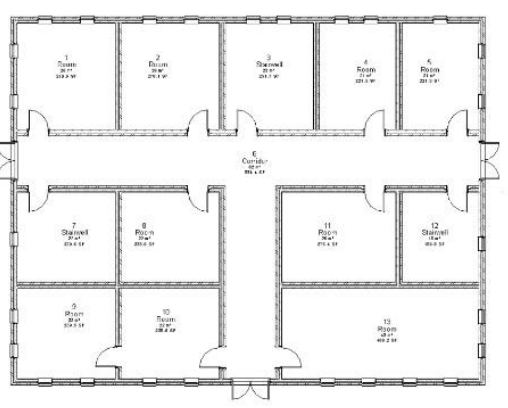

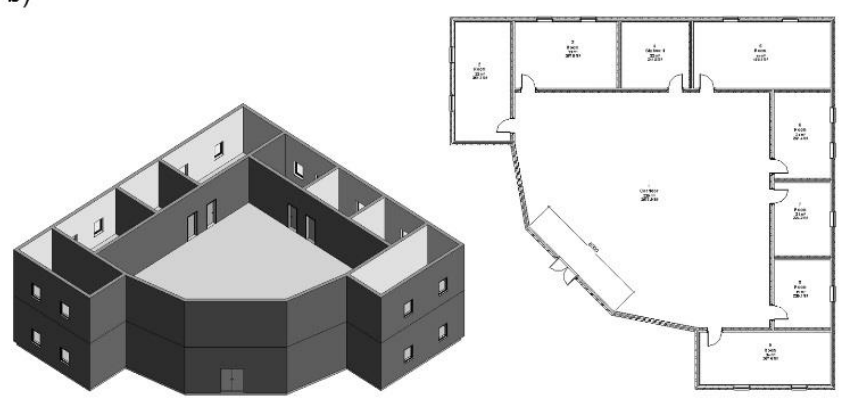

Figure 4. Mock data models - 3D views and ground floor plans: a) high-rise building; b) two-storey building.
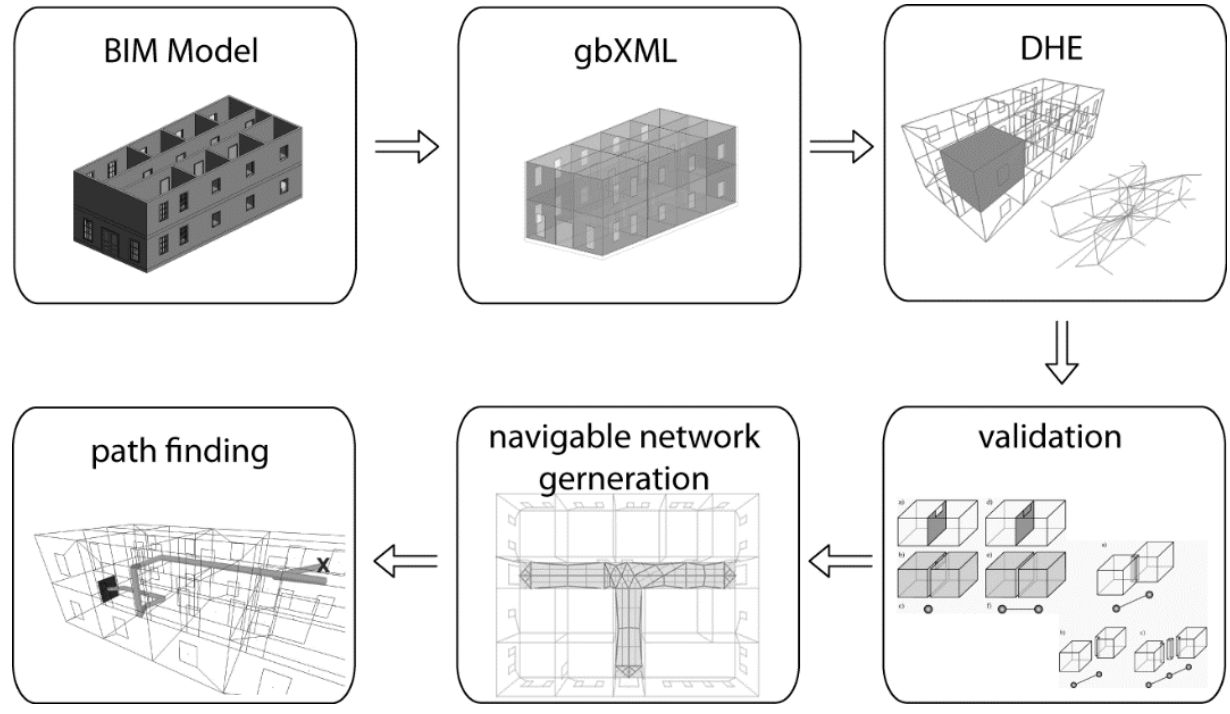

237

Figure 5. Method workflow.

The model enhancement, proposed in this paper, introduces a novel network for indoor navigation in spaces along navigation routes, such as corridors, big open spaces, rooms with several doors. A floor is tessellated using a VD construction algorithm, where doors and concave corners are initial Voronoi vertices (nucleation points), and additional points are added in order to create a denser tessellation. The nucleation points from adjacent cells are connected by links, thus forming a network, which is better suited for navigation, than the network without such innovation.

There are several advantages to this strategy: 1 ) path finding algorithms for the additional network give more accurate results within a single room; 2 ) the additional network is generated using a 2D algorithm and does not affect the original network representing the 3D topology of a building; 3 ) the same method may be applied for corridors and big open spaces of an arbitrary shape; 4) 'natural' paths for human movement are reflected in the network. 
The proposed method consists of several steps: A) spaces (we will call them rooms in the following description) with more than one door are selected; B) the floor of the selected room is detected based on semantic information from the original BIM model or, if this information is not available, then surfaces with the normal vector pointing upwards are considered as a floor; C) dual nodes representing doors are projected on the floor and together with concave vertices of the floor surface are selected as nucleation points for a tessellation algorithm - they are called 'constraint nucleation points' in the following description; D) the VD algorithm is performed iteratively and additional points are added; the nucleation points of neighbour cells are connected by links forming a network; E) the locations of additional nucleation points occurring on constraint edges are moved towards the geometric centre of the associated cell, while the original location of the constraint points is not changed.

The process of the VD construction (see Step $\mathrm{D}$ above) requires a detailed description. An iterative algorithm for the constraint VD was implemented based on the Green and Sibson algorithm (Green and Sibson, 1978), see Figure 6.
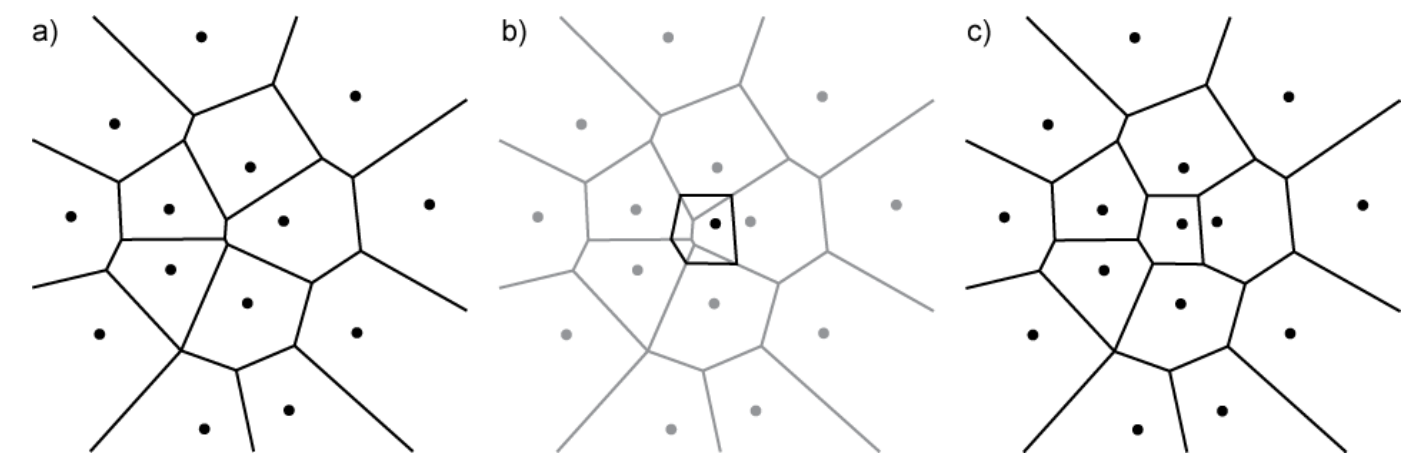

Figure 6. Process of Voronoi Diagram construction: a) existing tessellation; b) new vertex and its Voronoi cell; c) tessellation after point insertion.

The floor is represented by a polygon of an arbitrary shape. The original polygon edges are constraint edges, which are not modified in the tessellation process. In the first iteration dual nodes representing doors, projected on the floor and concave corners, are selected as constraint nucleation points. The locations of the constraint points are not changed later. The nucleation point is always enclosed by a single Voronoi cell. Subsequently, each node is connected by links with nodes from adjacent cells; all these links and nodes form a network. In the next step, new nucleation points are added at the middle of each link if the link is bounded by constraint points, or if both bounding points lie on constraint edges, which are not co-linear. The process is repeated until no new points may be added because they would be too close, or a certain number of iterations have been reached.

An algorithm for VD construction is presented below as Algorithm 1. In the examples presented in this paper, the following input parameters were used: the threshold $T_{1}=6$ (see Figure $7 c$ and Figure 8b), the threshold $T_{2}=0.7$ (see Figure 9) and the maximum tessellation level $T L_{\max }=10 . T_{2}$ is used if an edge $e$ in the tessellation is close to the hazard location. In this paper, $e$ is considered to be close to the hazard location if length $(e) \times 3>$ distance $(u, h)+$ distance $(v, h)$, where: $u$ and $v$ are end-points of $e$ and $h$ is the hazard location. 
For the sake of algorithm clarity, only one polygon representing a floor is considered. If a floor is represented as a set of adjacent polygons in an original model, they may be merged into one and the same algorithm can be used.

Algorithm 1: Navigable network generation (tessellation of a floor)

Input: The cell representing room $R$.

The floor polygon for $R$.

$T_{1}, T_{2}$ (threshold values for edge length).

Maximum tessellation level $\mathrm{TL}_{\max }$

\section{Output: Tessellation $f$.}

1. Make a copy of the floor polygon, denoted by $f$.

2. Calculate the number of doors in room $R$, denoted by $d$. If $d \leq 1$, then stop the algorithm (no network generation is necessary).

3. For each door dual node, make its projection on the boundary of $f$.

4. Create a list $N$ consisting of constraint nucleation points for VD in order of their appearance on the boundary of $f$. The constraint nucleation points are door nodes and concave corner nodes. $f$.

5. Create a list $V$ of VD cells and put $V_{1}=f$. (The floor polygon $f$ is the initial Voronoi cell associated with $N_{1 .}$.)

6. Put $T L=1$, where $T L$ is the current tessellation level.

7. Take the first unprocessed element from $N$, denoted by $N_{j}$.

8. Create a list of cells for testing, denoted by $C T$ and add to it a cell from $V$, which includes node $N_{j}$

9. Take the first unprocessed element from ctest and denote it by $V_{k}$ (it is associated with $N_{k}$ ).

10. Calculate a bisector line bi of the segment $\left(N_{j}, N_{k}\right)$.

11. Calculate intersection points of $b i$ with $V_{k}$. The set of such points is denoted by IP.

12. If the number of points in $I P$ is greater than 2 , then do the following:
a. In I find two intersection points whose line segment divides the cell $V_{k}$ into two parts such that each part includes one of the nodes $N_{j}$ and $N_{k}$;

b. Remove the rest of the intersection points from IP.

13. If the number of points in $I P$ is less than 2 , then go to Step 9.

14. Create the edge $(j, k)$ bounded by intersection points from $I P$, and insert it into the existing tessellation of the polygon $f$. The edge $(j, k)$ divides $V_{k}$ into two cells: $C_{j}$ and $C_{k}$ such that $N_{j}$ is located in $C_{j}$ and $N_{k}$ in $C_{k}$.

15. Add $C_{j}$ to $V$, i.e. $V_{j}=C_{j}$, and put $V_{k}=C_{k}$. (The edge $(j, k)$ is a part of the boundary of the newly created cell for $N_{j}$.)

16. Add those cells to the list $C T$, which are adjacent to the edges intersected by the edge $(j, k)$, taking into account the following:

a. If an intersected edge is a constraint edge (the initial boundary of $f$ ), then add all cells adjacent to $V_{k}$.

b. If a cell is already in $C T$, then do not add it.

17. If there are unprocessed elements in $C T$, then go to Step 9. (Otherwise the boundary of the new cell for $N_{j}$ was created.) 
18. Remove all edges from inside the boundary of the newly created cell for $N_{j}$. (This is illustrated in Figure 6 , the transition from b) to c).)

19. If there are unprocessed elements in $N$, then go to Step 7. (Otherwise the current level of tessellation is completed.)

20. If $T L<T L_{\max }$, then for each edge $e$ connecting nucleation points of adjacent cells in $v d$, do the following. Calculate the bisector point $B$ for $e$ only if:

a. $e$ is longer than $\mathrm{T}_{1}$; or

b. $e$ is close to the hazard location and it is longer than $\mathrm{T}_{2}$; or

c. $e$ is bounded by two constraint nucleation points (doors or concave corners); or

d. both end-points of $e$ lie on constraint edges, which are not co-linear.

Add the point $B$ into $N$.

21. If $T L<T L_{\max }$ and there are unprocessed elements in $N$, then $T L=T L+1$ and go to Step 7 .

22. If there are nucleation points in $n p$, other than constraint nucleation points, which are located on constraint edges (the initial boundary of $f$ ), then move locations of those points towards the centre of the associated cells.

23. Report $f$. Algorithm stops.

The first iteration of the tessellation (grey cells) and the corresponding network (black edges) are shown in Figure 7a. Because the tessellation is not dense, the network consists of few links. Many of them lie on constraint floor edges. Therefore, several navigation routes go from door to door along walls. The process stops after the second iteration generates shorter links (see Figure 7b). Shorter links are the result of new point insertion in the midpoint of the original link, which leads into generation of a Voronoi cell with associated dual edges (i.e. shorter links) connecting the new cell with neighbour cells. The locations of new nucleation points occurring on constraint edges are modified by moving them towards the centre of the associated cell (see Step E), which overcomes the problem of navigation along walls. However, there are some links in the network, which are perpendicular to the walls, and their bounding points occur on constraint edges. Such links may produce sharp turns on navigation routes. Additional nodes and links are added in the next iterations, which solves this problem. The final result is shown in Figure 7c. The tessellation and corresponding network are attached to the dual node representing the room. The network is used for precise path calculation within a room. 

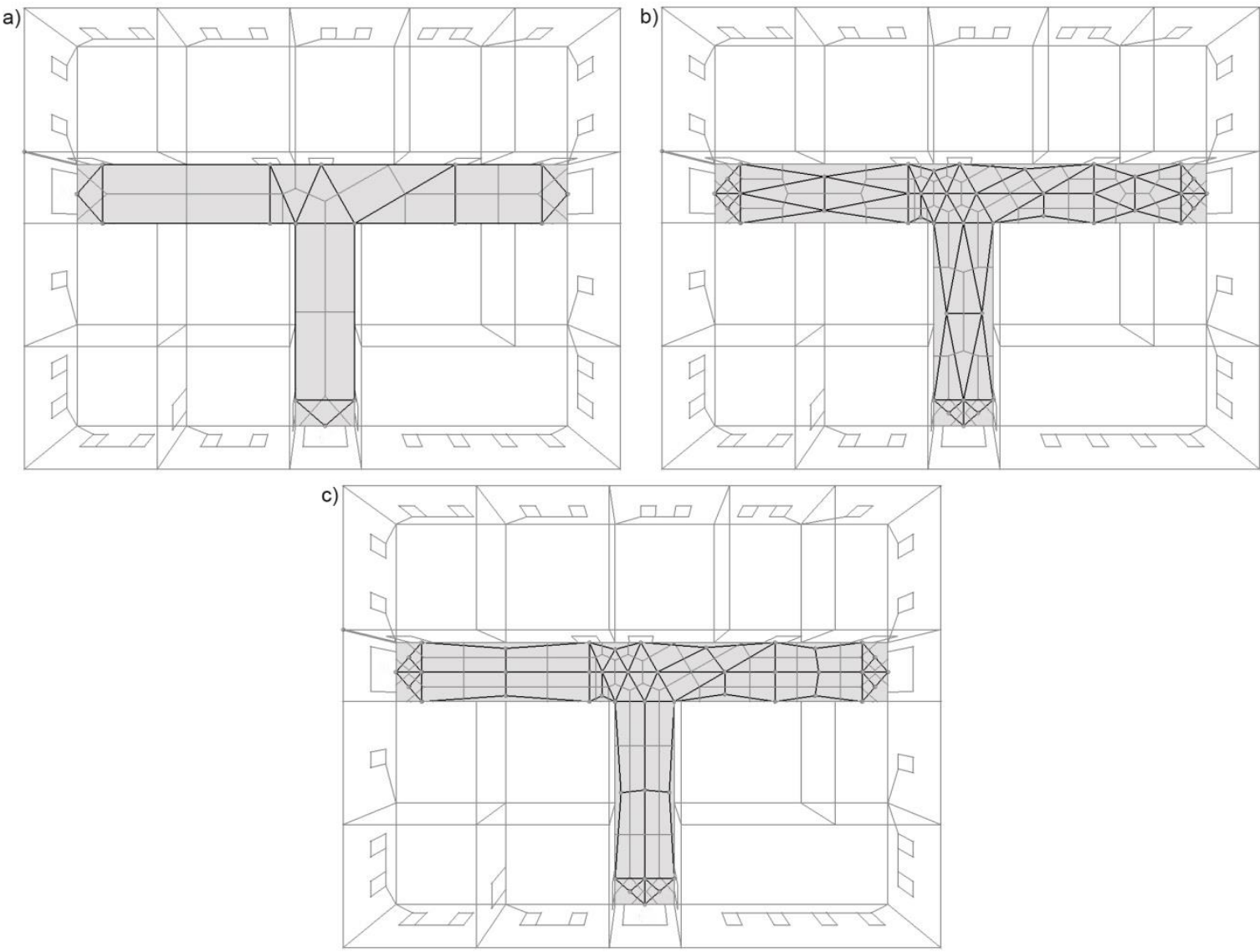

Figure 7. Floor tessellation (grey cells) and associated network (black lines): a) first iteration; b) second iteration; c) final tessellation.

An advantage of the proposed solution is that it can be applied not only to corridors but also to any room shape, e.g. irregular spaces. This is an important property, which helps to avoid the need to classifying a room as a corridor or an open space, and then performing different algorithms for network generation. An example of an indefinable area is a narrow corridor which, at some point, turns into a bigger area (a hub) with many doors or a junction with other corridors.

However, in this scenario, long links in the network may be introduced, because nucleation points in the central area of a room are enclosed by relatively bigger cells and the distance to neighbours is bigger (see Figure 8). This may produce 'wobbling' paths. Consequently, for links that are longer than a specified threshold, new nucleation points are introduced in the midpoint of these links. 
a)

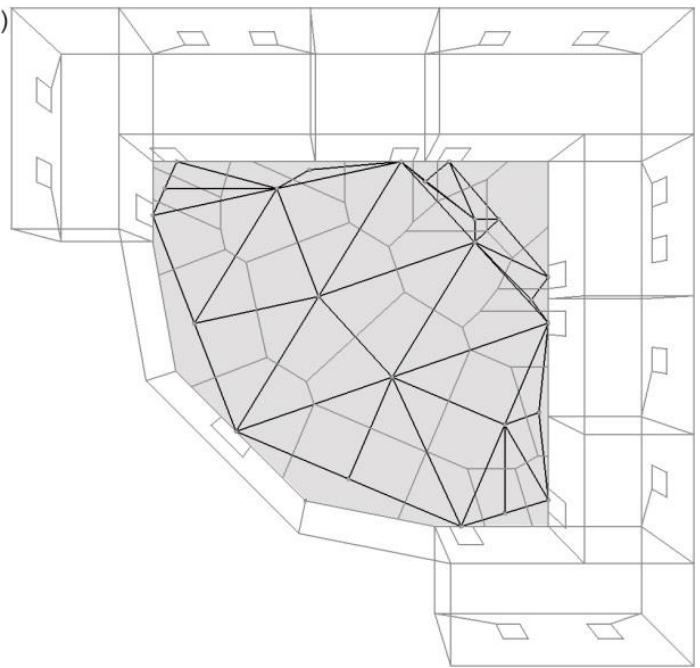

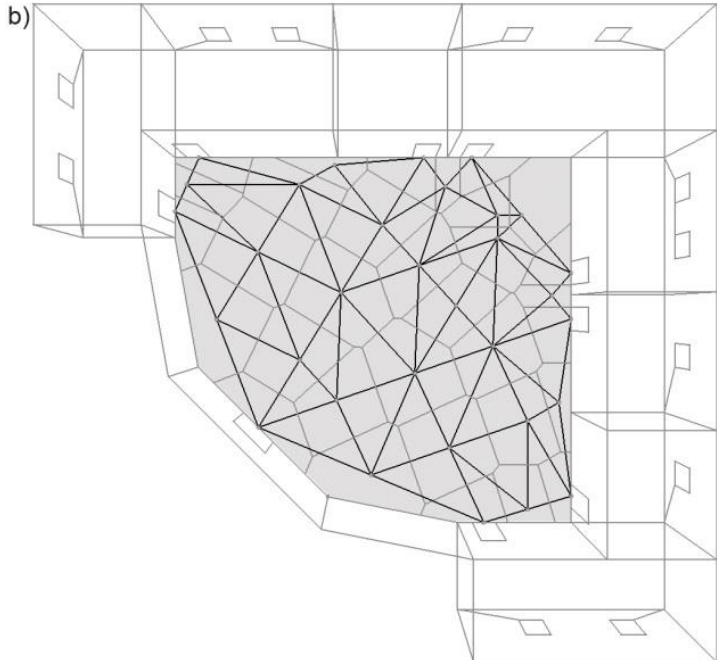

Figure 8. Tessellation of big open space (grey area): a) tessellation without a maximum link length threshold; b) tessellation with a maximum link length threshold (in this case $6 \mathrm{~m}$ ).

The higher density of the network is helpful for finding alternative or safest paths when a hazardous event takes place within an open space: some nodes may be prohibited for navigation, while the rest of the space, with a lower risk, is still considered as safe. This applies to buildings such as airports or shopping malls.

The network might be densified around a dangerous area using the same method. For links located closer to the hazard source, a smaller threshold for links is introduced. Therefore, new nucleation points are introduced in the midpoints of these links and new shorter links are introduced. This operation provides local network densification (see Figure 9). TODO: (move to introduction???) Densification is commonly applied in the finite element mesh generation and refinement (Du and Gunzburger, 2002; Ho-Le, 1988; Petersen and Martins, 1997).

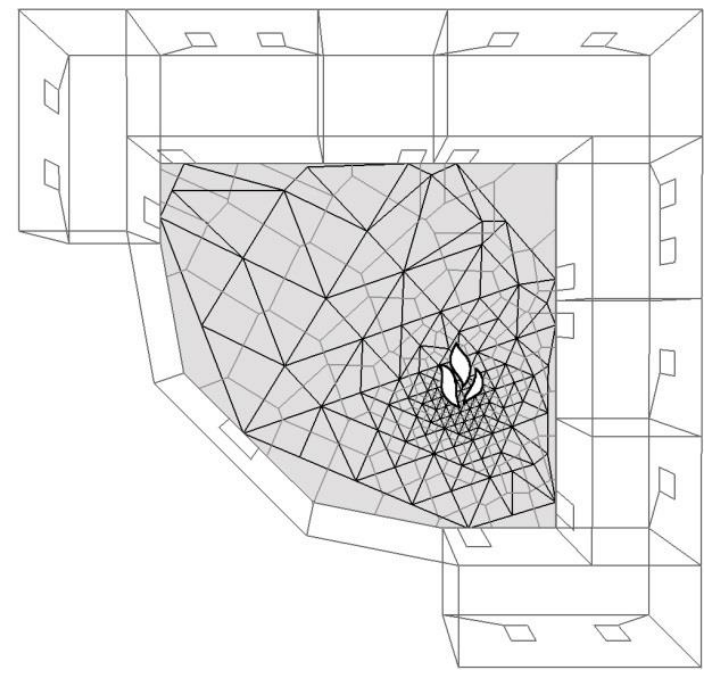

Figure 9. Local densification of the navigable network around a hazardous area.

\subsection{Results and discussion}

The main reason for introducing additional nucleation points is to improve the network for a path finding application. This increases the number of cells in the tessellation, thus the number of 
network links is increased and they are shorter. If the tessellation process is finished after the first iteration, a path between two locations goes through constraint points, which is not a 'natural' route for navigation - it is like walking from door to door along walls (see Figure 10a). The second iteration produces a network better suited for navigation, but the path is slightly 'wobbling' (see Figure 10b), mainly because there are links that cross the corridor - perpendicular to the constraint edges. The path based on the final network is still influenced by 'wobbling' but less than in the previous iterations (see Figure 10c), and it is acceptable in the presented research, since a very precise path calculation is not required.

a)

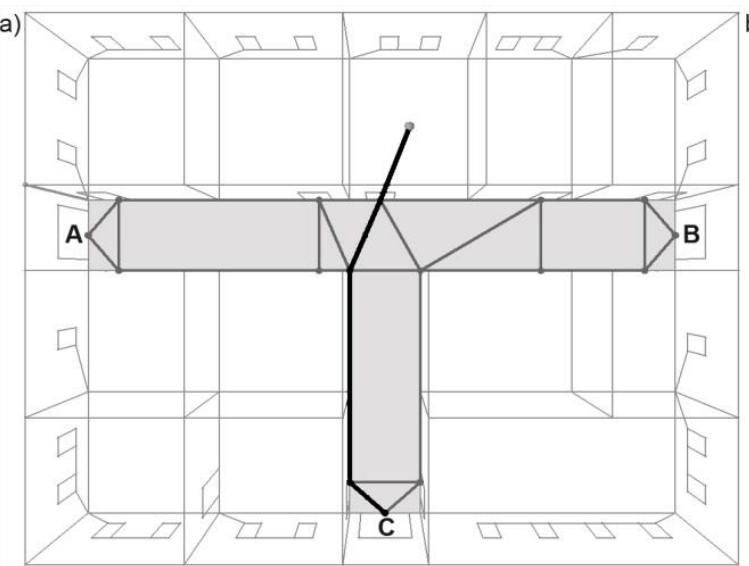

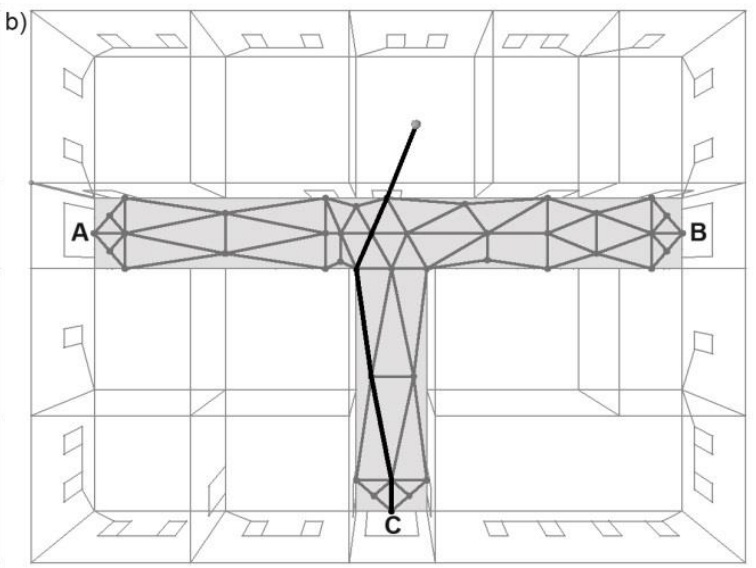

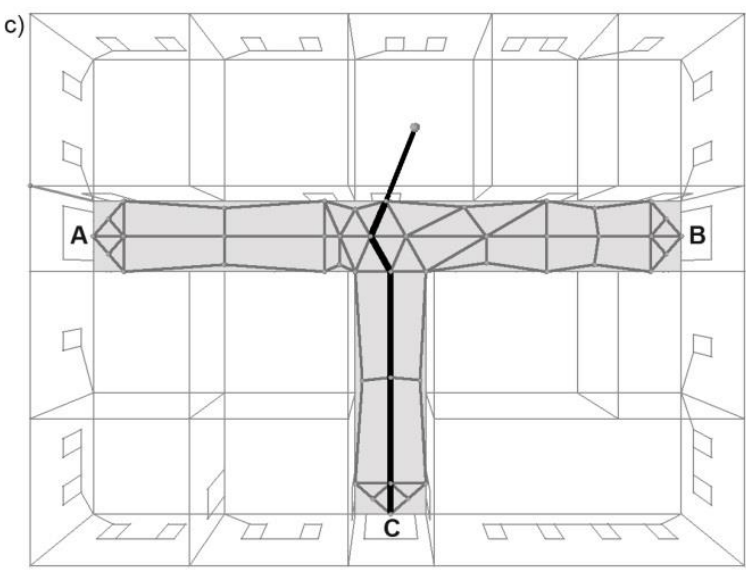

Figure 10. Shortest path from a selected room to door C: a) using the network produced by the first iteration; b) using the network produced by the second iteration; c) using the final network.

The proposed method solves the problem of incorrect paths shown in Figure 3. When the additional network within the corridor is used, the result of the shortest path finding algorithm is correct (see Figure 11). The egress route from the selected room goes through the door straight to the nearby exit. 


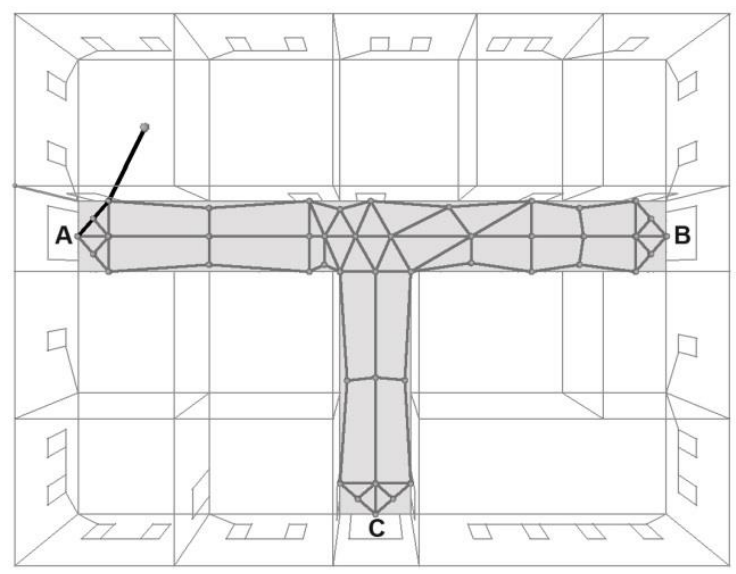

Figure 11. The shortest path from a selected room to exit A (A-C are exits from a building) based on 404 the original network and the network produced by the corridor floor tessellation.

A satisfactory improvement is also achieved in the case of open spaces. All shortest paths from a selected room to all doors of the open space are shown in Figure 12. The network within the room is a better approximation of 'natural' navigation routes than the original logical network.

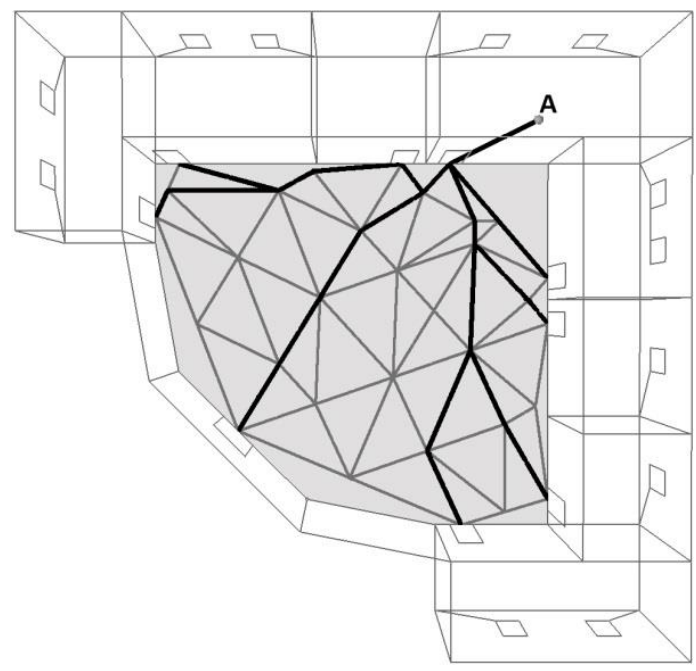

Figure 12. Shortest paths (black lines) from a selected room (A) to all doors of an open space.

The result of Dijkstra's algorithm applied from a selected room in the building is shown in Figure 13. The shortest path goes from the room at the top floor to the ground floor through one of three staircases located near the room. All corridors and rooms with more than one door in the building were automatically tessellated and the navigable network was retrieved before the application of the path finding algorithm.

It should be noted that the building model used here is a mock dataset, where the main navigation routes include corridors and staircases, and lifts are not included in the model. However, lift shafts may be considered for navigation in the same way as staircases but normally they cannot be used in case of emergency situations. 


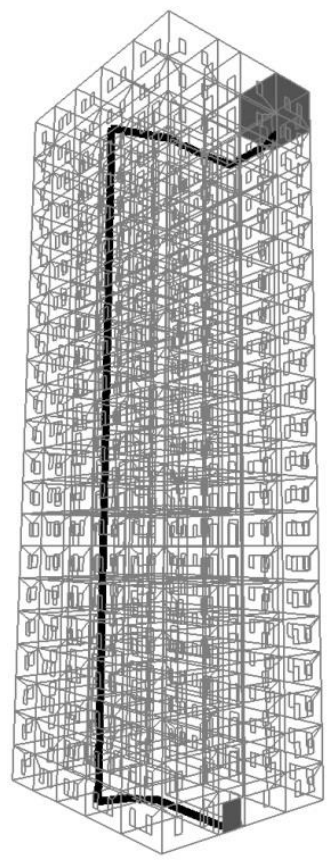

420 Figure 13. Egress route (black lines) from a selected room (grey cell) to the closest exit (grey door).

421 The logical network may be used for alternative path finding in the case of an emergency, when the 422 conventional navigation routes are blocked. In the hypothetical scenario shown in Figure 14, a 423 section of the corridor may be on fire. Therefore, it is not safe to use a conventional route to leave 424 the selected room and reach exit $A$ as a result of a fire behind the door. Thus, an alternative path is 425 calculated, which goes through walls, where a hole can be possibly made. Once the room with a 426 door located outside the dangerous area is reached, a conventional egress route to exit $B$ is used.

427 Alternative paths are found automatically if there is no safe connection from a room through doors. 428 A connection from the logical network with the smallest weight is selected. The weight for each wall 429 in the room may differ depending on a construction material. Therefore, walls easier to demolish are 430 selected as candidates for navigation, e.g. partitions made of plaster panels is favoured over walls 431 made of concrete.

432 It is important to note that windows may be considered as alternative exits if they are located close 433 above the external ground, for example, windows on the ground floor. In addition, rescue teams are 434 able to get inside the building through windows located high above the ground using ladders. In 435 order to show navigation through walls using the logical network windows were not allowed to be 436 used in the illustrated simulation. 


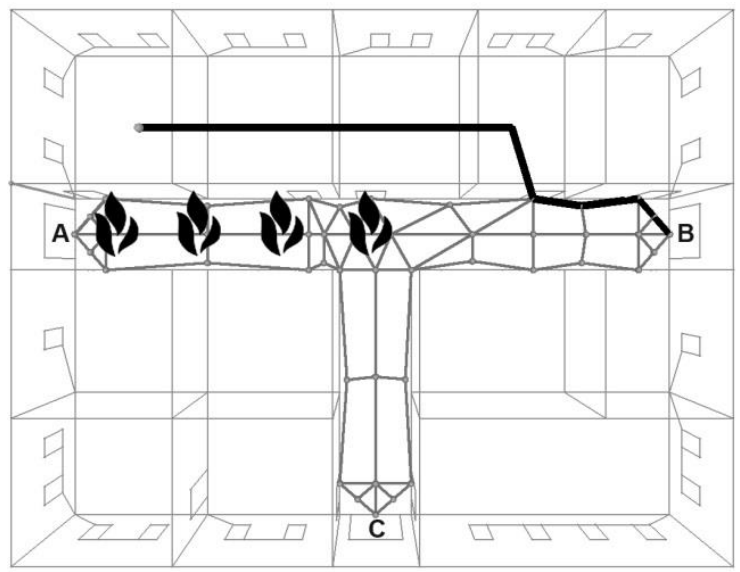

Figure 14. Alternative path finding.

Another type of alternative paths are the safest paths, which avoid dangerous areas using normal navigation ways. Typically they are longer than shortest paths but they are located as far from hazard as possible. In current research, an abstract hazard is manually triggered in selected rooms and is propagated within a building. If information from sensors (e.g. temperature and smoke sensors) is available, a hazardous event may be automatically detected. In hazard simulation, additional weights reflecting hazard spread in the network are calculated based on the number of obstacles, such as walls, and distance from hazard locations. Then, new safest paths are calculated using these new weights.

\subsection{Comparison of VDN with prevailing indoor navigable networks}

Two state of the art methods of the navigable network reconstruction used in emergency response research (Lee, 2004; Liu and Zlatanova, 2011) were selected for a comparison. The reason for this selection is the close relationships to the VDN method proposed in this paper. Three configurations of rooms shown in Figure 7, Figure 8 and Figure 15 are used as examples for this comparison: Tshaped corridor (also see Figure 16 a), d), g) and j)), open space (also see Figure 16 b), e), h) and k)) and Z-shaped corridor (also see Figure $16 \mathrm{c}$ ), f), i) and I)), respectively. Two first examples are mock data, while the latter is a real building - World Trade Centre in Doha, Qatar.

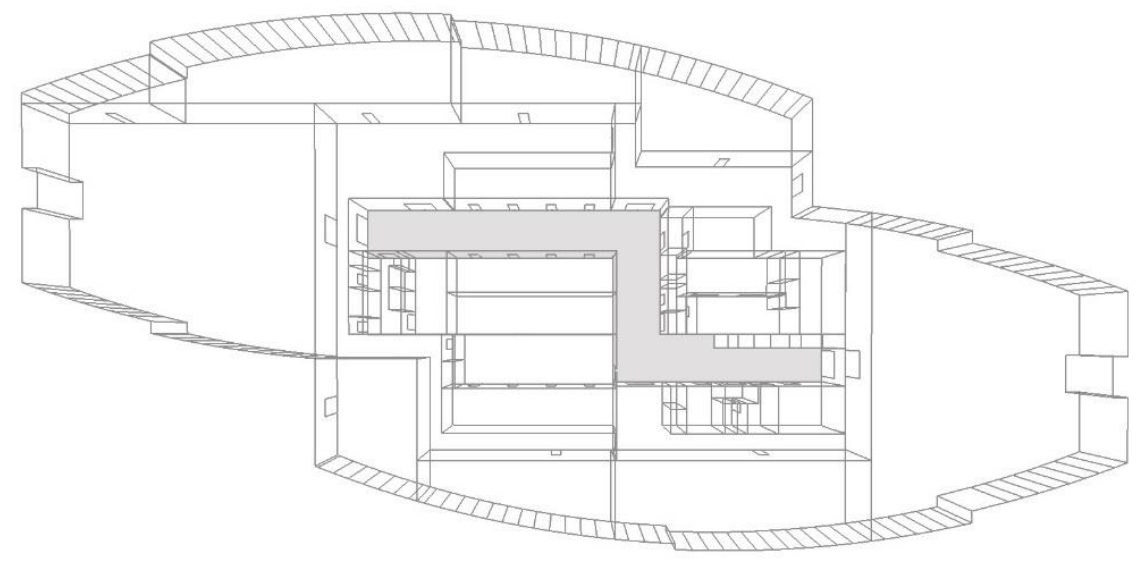

Figure 15. Floor plan - World Trade Centre in Doha, Qatar. Grey-shaded corridor is used for comparison with other methods. 
458 Lee (2004) proposed the Node-Relation Structure (NRS) as the topological model. The Straight

459 Medial Axis Transformation is applied for generating a navigable network within a corridor. A

460 skeleton of the corridor floor is calculated; this is based on VD construction. The result is a linear

461 representation of a polygon - a 'backbone' representing a navigation route through the corridor.

462 Nodes representing rooms adjacent to the corridor with a door in between are connected to the

463 skeleton by additional links. The advantage of this method is that it is a simple network with a

464 relatively small number of links (see Table 1 ).

465 However, the applicability to big open spaces was not presented, possibly because of unsatisfactory

466 representation of navigation routes. Consequently, it is suggested that it is not practical to use the

467 aforementioned method for spaces other than simple-shaped corridors. Additionally, because the

468 skeleton is located in the middle of the corridor and the adjacent rooms are connected to the

469 skeleton by perpendicular links, the network does not reflect 'natural' ways of navigation - if two

470 doors are coplanar, i.e. located on the same wall, one must go from the first door to the middle of a

471 corridor, turn 90 degrees, walk in the middle of the corridor towards the second door, turn 90

472 degrees again, and then get to the second door. This may be not a significant drawback, especially

473 for narrow corridors when the shortest paths are calculated. However, as complexity increases, it

474 may be become significant in computation of the simplest paths, where the sharpness of turn angle

475 is considered.

476 The door-to-door (DtD) method (Liu and Zlatanova, 2011) is based on a simple idea of navigation

477 from a selected door directly to another door, if two doors are in direct visibility. If there is no direct

478 visibility, then intermediate concave corridor corners are introduced to the path. Taking all possible

479 connections among all the doors within a corridor including concave corners, the navigable network

480 consists of more links than NRS (see Table 1). The network is a non-planar graph in the considered

481 examples. The method may be easily applied for big irregular spaces.

482 These two methods produce a fixed network depending on the geometry of a room and location of

483 doors. This may be a drawback when alternative paths within a big room are necessary to calculate,

484 for instance to avoid hazard located in the room. This is critical, when big open spaces in a shopping

485 mall or airport are considered. In contrast, the proposed VDN method is more flexible, mainly

486 because the density of tessellation may be easily increased resulting in more links in the network

487 and thus more flexible routes (see Table 1). 


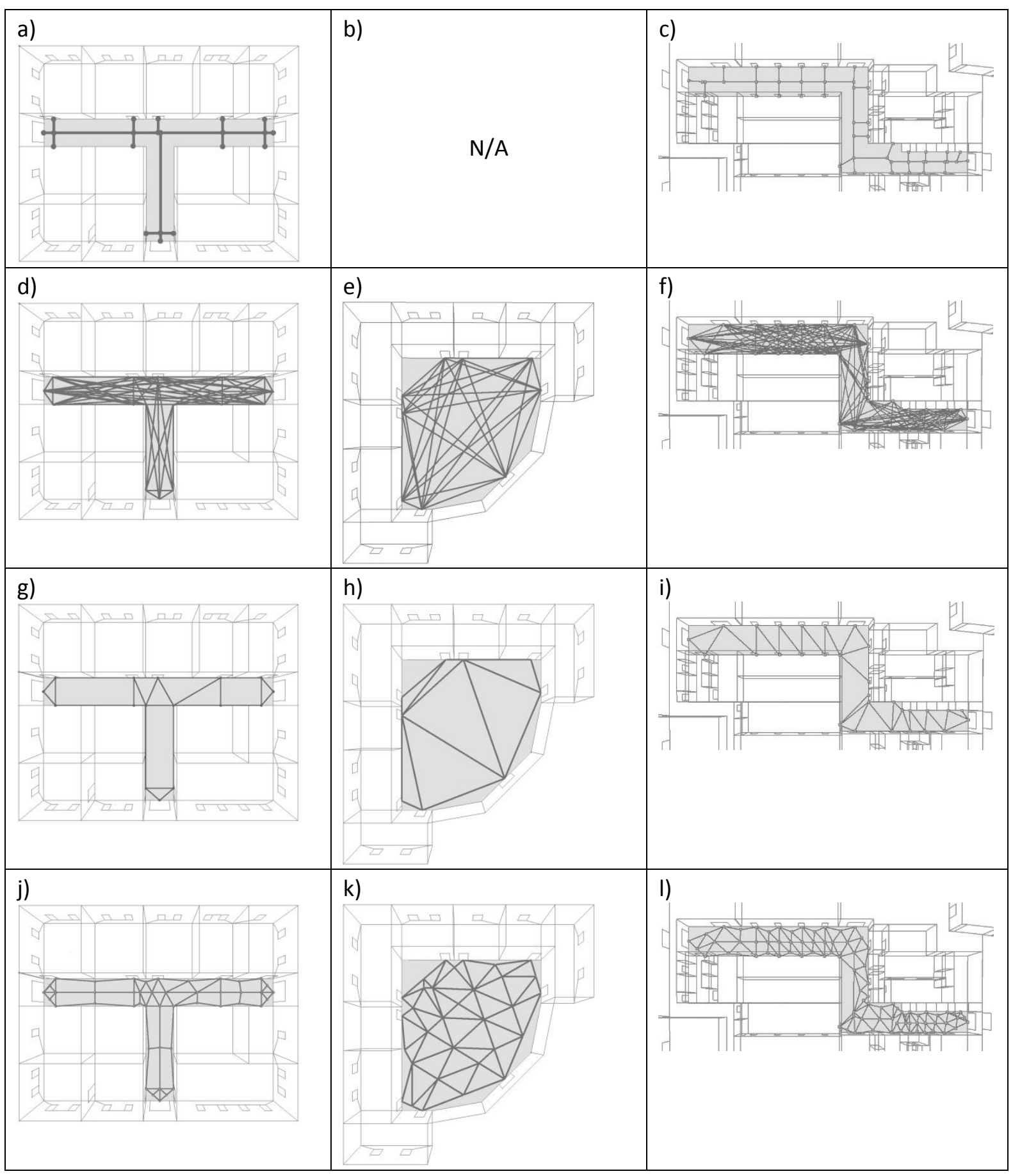

Figure 16. Navigable networks generated for different floor shapes using different methods: a)-c) NRS; d)-f) DtD; g)-i) VDN (1 ${ }^{\text {st }}$ level); j)-I) VDN (max. level with the threshold).

490 It should be noted, that the number of links calculated for the DtD method is based on the following assumption: links connecting the same nodes are not duplicated in the network. For example, considering three doors $d_{1}-d_{3}$ and one concave corner $c_{1}$, if doors $d_{1}$ and $d_{2}$ are in a direct visibility and $d_{3}$ is connected with $d_{1}$ and $d_{2}$ through $c_{1}$, there are four links: $d_{1}-d_{2}, d_{1}-c_{1}, d_{2}-c_{1}$ and $c_{1}-d_{3}$.

It should be also noted that, in the NRS construction, the skeleton of the corridor is calculated based on straight medial axis transformation. Door nodes are connected to the skeleton by line segments, which are perpendicular to the skeleton, and new nodes at an intersection point are added to the skeleton. However, if the distance from a door to an existing node in the skeleton is shorter than the 
perpendicular connection, the door node is connected to that node. Conversely, in order to avoid very short links in the network, a door node is connected to an existing node if a new intersection point is located within a range of $10 \mathrm{~cm}$ from the existing node.

501 Table 1. Number of nodes and links in navigable networks generated using different methods.

\begin{tabular}{|c|c|c|c|c|}
\hline & $\begin{array}{c}\text { NRS } \\
\text { (Lee, 2004) }\end{array}$ & $\begin{array}{c}\text { DtD } \\
\text { (Liu and } \\
\text { Zlatanova, 2011) }\end{array}$ & $\begin{array}{c}\text { VDN } \\
\left(1^{\text {st }} \text { level }\right)\end{array}$ & $\begin{array}{c}\text { VDN } \\
\text { (max. level with } \\
\text { the threshold) }\end{array}$ \\
\hline $\begin{array}{l}\text { T-shaped } \\
\text { corridor }\end{array}$ & $\begin{array}{l}20 \text { nodes } \\
20 \text { links }\end{array}$ & $\begin{array}{l}16 \text { nodes } \\
77 \text { links }\end{array}$ & $\begin{array}{l}16 \text { nodes } \\
26 \text { links }\end{array}$ & $\begin{array}{l}45 \text { nodes } \\
84 \text { links }\end{array}$ \\
\hline $\begin{array}{l}\text { Open } \\
\text { space }\end{array}$ & $\mathrm{N} / \mathrm{A}$ & $\begin{array}{l}9 \text { nodes } \\
36 \text { links }\end{array}$ & $\begin{array}{l}9 \text { nodes } \\
15 \text { links }\end{array}$ & $\begin{array}{l}32 \text { nodes } \\
74 \text { links }\end{array}$ \\
\hline $\begin{array}{l}\text { Z-shaped } \\
\text { corridor }\end{array}$ & $\begin{array}{c}47 \text { nodes } \\
46 \text { links }\end{array}$ & $\begin{array}{l}30 \text { nodes } \\
187 \text { links }\end{array}$ & $\begin{array}{l}30 \text { nodes } \\
56 \text { links }\end{array}$ & $\begin{array}{l}85 \text { nodes } \\
190 \text { links }\end{array}$ \\
\hline Comments & $\begin{array}{l}\text { - simple network } \\
\text { with a small } \\
\text { number of links } \\
\text { - limited } \\
\text { applicability to } \\
\text { open spaces }\end{array}$ & $\begin{array}{l}\text { - non-planar } \\
\text { graph } \\
\text { - minimized } \\
\text { number of links } \\
\text { on door-to-door } \\
\text { routes }\end{array}$ & \multicolumn{2}{|c|}{$\begin{array}{l}\text { - variable number of links depending on } \\
\text { densification level } \\
\text { - local network densification possible }\end{array}$} \\
\hline
\end{tabular}

502

503

504

505

506

507

508

509

510

In order to compare the precision of different methods for tessellation, we consider the main corridor of the actual floor plan of the Doha World Trade Centre (see Figure 15). There are 27 doors in the corridor as depicted in Figure 17. Ten pairs of doors were randomly generated, which represent ten random possibilities for moving from one door to another in the corridor (see Table 2). For each pair of doors, the length of the actual walking path between them was calculated, as well as the lengths of paths generated by the NRS method, the DtD approach and the VDN method for the first and maximal levels of tessellation. The corresponding results are summarised in Table 2. Examples of path $P_{6}$ generated by the aforementioned methods are shown in Figure 18.

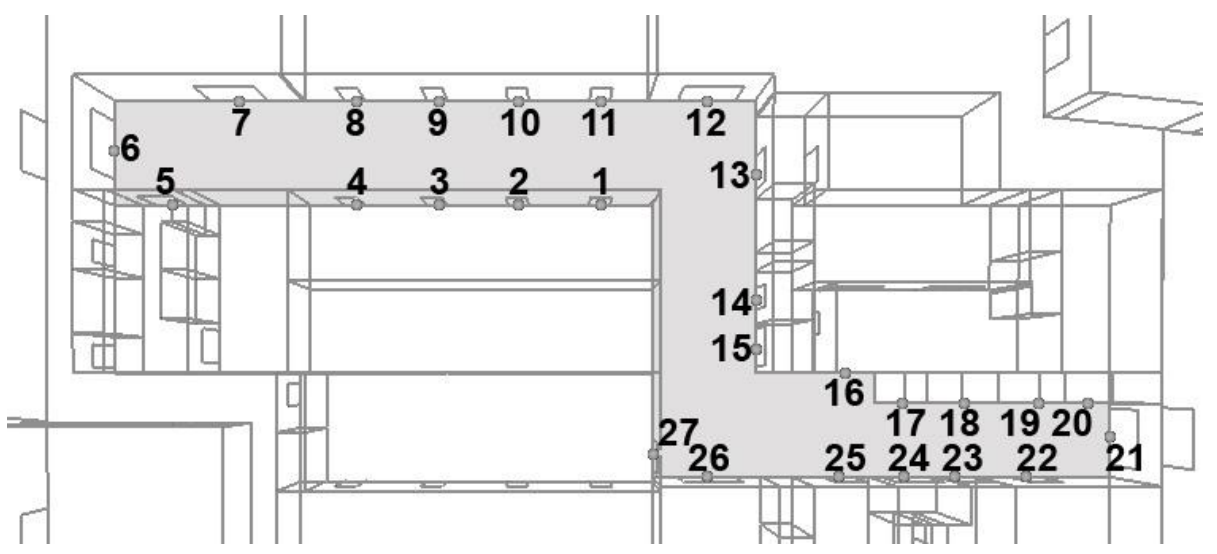

Figure 17. Location of doors 1-27. 
Table 2. Ten random routes and their lengths (meters) for different methods

\begin{tabular}{|c|cc|ccccc|}
\hline Path & $\begin{array}{c}\text { From } \\
\text { Room }\end{array}$ & $\begin{array}{c}\text { To } \\
\text { Room }\end{array}$ & Actual Route & NRS & DtD & $\begin{array}{c}\text { VDN } \\
\text { (1 }^{\text {st level) }} \text { ) }\end{array}$ & $\begin{array}{c}\text { VDN } \\
\text { (max level } \\
\text { with the } \\
\text { threshold) }\end{array}$ \\
\hline$P_{1}$ & 11 & 23 & 18.8 & 24.1 & 18.0 & 19.3 & 19.5 \\
$P_{2}$ & 7 & 9 & 7.4 & 10.1 & 6.6 & 6.6 & 6.8 \\
$P_{3}$ & 5 & 16 & 28.1 & 34.3 & 25.4 & 26.0 & 27.3 \\
$P_{4}$ & 17 & 12 & 15.7 & 18.8 & 14.1 & 14.8 & 16.1 \\
$P_{5}$ & 15 & 20 & 14.1 & 18.1 & 12.0 & 12.2 & 13.2 \\
$P_{6}$ & 25 & 8 & 22.7 & 28.3 & 21.4 & 23.1 & 23.1 \\
$P_{7}$ & 10 & 26 & 15.7 & 18.7 & 14.8 & 17.1 & 15.6 \\
$P_{8}$ & 20 & 18 & 5.1 & 6.2 & 4.1 & 4.1 & 4.2 \\
$P_{9}$ & 9 & 1 & 6.8 & 8.9 & 6.4 & 7.1 & 6.6 \\
$P_{10}$ & 22 & 2 & 22.9 & 29.0 & 20.6 & 22.0 & 22.6 \\
\hline
\end{tabular}

514

a)

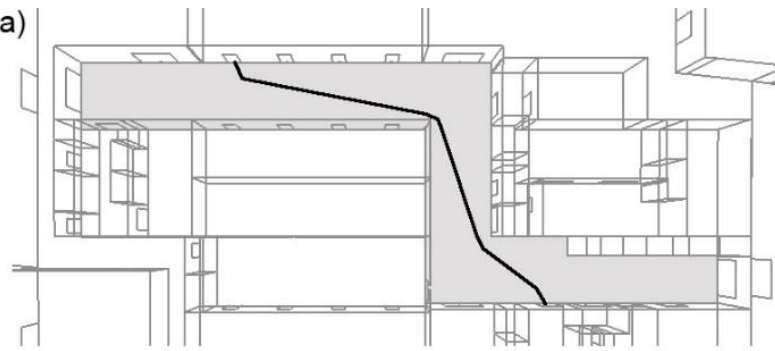

c)

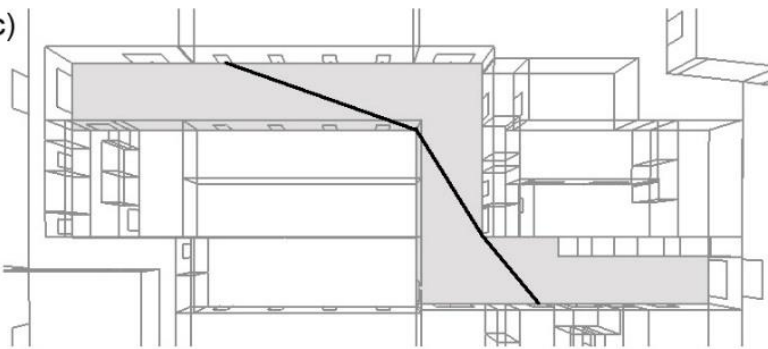

b)

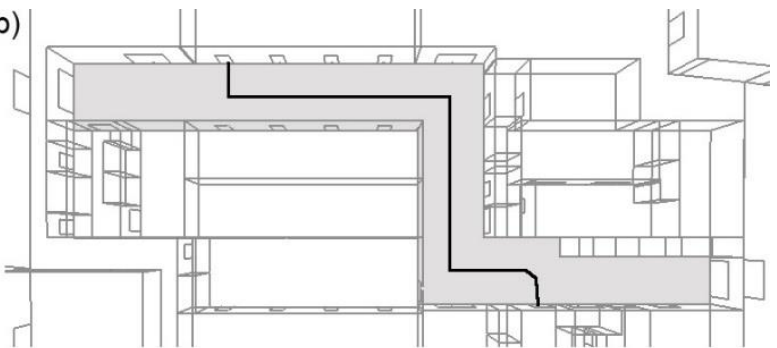

d)

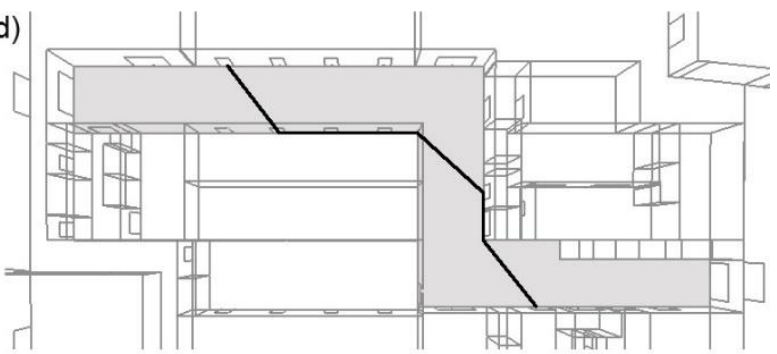

e)

Figure 18. Path $\mathrm{P}_{6}$ from room 25 to 8 generated by methods: a) actual walking path; b) NRS; c) DtD;

d) VDN (1 $1^{\text {st }}$ level); e) VDN (max level with the threshold)

518 The actual path, which is a point of reference in this comparison, was generated manually 519 considering the following rules: the path should not run closer than $0.8 \mathrm{~m}$ to the concave corners 520 (see Figure 19a); a next node after a door node is located $0.8 \mathrm{~m}$ from the door frame into direction 521 of the next move (see Figure 19b); the direction of door opening is not taken into consideration. 
a)

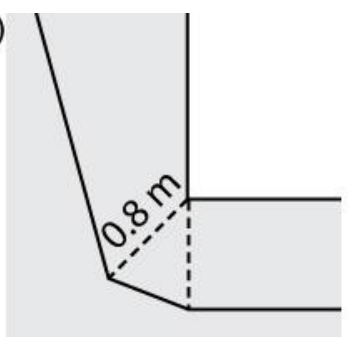

b)

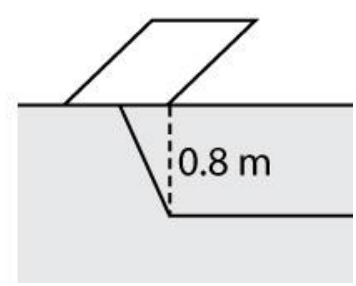

Figure 19. Actual path generation: a) distance from a wall; b) distance from a door.

Furthermore, three standard errors were calculated: the mean error (ME), the mean absolute error (MAE) and the mean squared error (MSE). Note that the former represents the bias, whereas the last two refer to the accuracy of methods. The results are given in Table 3. The NRS method very often overestimates the length of the actual path, which is reflected in the ME equal to $-3.9 \mathrm{~m}$, whereas the DtD approach tends to underestimate the length with $M E=1.4 \mathrm{~m}$. On average, there is some underestimation in the VDN method with the ME $=0.5 \mathrm{~m}$ and $0.2 \mathrm{~m}$ for the first and maximal levels. The MAE figures for the above methods are similar: $3.9 \mathrm{~m}, 1.4 \mathrm{~m}, 1.0 \mathrm{~m}$ and $0.5 \mathrm{~m}$, respectively. The same numbers expressed as percentages of the MAE to the average length (15.7 $\mathrm{m}$ ) of actual routes are as follows: $24.8 \%, 8.8 \%, 6.4 \%$ and $3.3 \%$, thus the $5 \%$ standard threshold for errors is achieved by the maximal level of tessellation in the VDN method. Finally, the MSE measure, which penalises large errors, is rather high (18.1) for the NRS method, whereas the DtD and VDN approaches exhibit small MSEs as can be seen in Table 3. Thus, the VDN method with the maximal level of tessellation demonstrates the best characteristics for the bias and accuracy.

Table 3. Errors of the four methods

\begin{tabular}{|c|cccc|}
\cline { 2 - 5 } \multicolumn{1}{c|}{} & NRS & DtD & VDN (1st level) & VDN (max level) \\
\hline ME & -3.9 & 1.4 & 0.5 & 0.2 \\
MAE & 3.9 & 1.4 & 1.0 & 0.5 \\
MSE & 18.1 & 2.4 & 1.3 & 0.4 \\
\hline
\end{tabular}

\subsection{Conclusions}

In this paper, the navigable indoor network reconstruction from an indoor model was presented and compared with other methods applied for route planning in the field of emergency preparedness and response. The network consists of a logical network reflecting the 3D building structure and a detailed navigable network within rooms with many doors and complex geometry. The logical network represents the full 3D topology including spatial relations between rooms at different floors and rooms sharing a wall without doors or other openings. It can be used not only for indoor navigation but also for alternative path finding and potential simulations of various phenomena associated with emergency situations.

DHE data structure was used in this research to represent a 3D indoor scene reconstructed from a BIM model. Two graphs, primal and dual, stored and updated simultaneously allow for the geometry, topology and semantics representation in one consistent model. They are used for direct implementation of Dijkstra's algorithm for path-finding and visualisation of the model, and egress routes. 
552 To demonstrate the robustness of the VDN approach, a static model was presented. This consists of

553 a structure of the building and location of events that are known before the navigable network is

554 generated. In case of a new event, the whole navigable network within an affected room is

555 generated from the start. However, local densification of the network may be performed, when

556 necessary, without recalculating the whole network.

557 The VDN algorithm may be applied to any space type including corridors and big open spaces, unlike

558 other methods, which focus on corridors only. The tessellation may be easily densified locally or 559 within the whole space by changing the threshold for a link length, which increases the number of

560 links in the navigable network. This may be helpful in finding alternative paths within a single big

561 room with a hazardous event located within the room. Because VDN is generated independently for

562 each space, a different tessellation level and threshold may be defined for different spaces, for

563 example, denser network available within corridors, while sparse in rooms.

564 It was shown that VDN, in comparison to prevailing approaches, increases accuracy of egress route

565 planning. The length of paths calculated using VDN is closer to the length of 'natural' paths taken by

566 pedestrians. Comparison study was performed in a model of the real building: Doha World Trade

567 Centre in Qatar.

568 The proposed method uses basic algorithms for network construction (Green and Sibson algorithm

569 for VD generation), densification (new nucleation points added in the middle of links longer than a

570 threshold) and path-finding (Dijkstra's algorithm) in order to present a general idea and its

571 applicability in emergency response applications. It may be improved by using advanced algorithms

572 for better Voronoi point location, e.g. using Lloyd's algorithm (Du et al., 1999), real-time network

573 updates including point deletion (Mostafavi et al., 2003) and network densification utilized in finite

574 element mesh generation. These wo-operations, i.e. change of nucleation point location and

575 network densification, mayshould improve effectiveness of the proposed method by quick re-

576 computation of the network and generation of more natural the-shape of for navigable pathsin

577 such a way that more natural paths are generated. Enhancement of the navigable network in order

578 to getobtain natural paths and quick network updates will be considered in future research.

579 Methods of alternative path-finding are currently under development in presented research. An

580 alternative path may be understood in two ways: i) a path, which runs through a wall if all standard

581 navigation routes through doors are blocked; ii) a path, which is longer than the shortest path and

582 runs through standard navigation routes, but avoiding hazardous areas. In future work it is planned

583 to include information about construction material in models and use it for more advanced egress

584 route finding and simulations of hazard propagation in buildings.

585 The future work includes development of the decision support system based on VDN and the multi-

586 attribute decision-making technique, which takes shortest, safest and simplest paths as input data

587 and returns 'the best path'. The system will be suited for rescue andemententereams, who need to

588 get access to trapped people. 
This research/publication was made possible by a National Priority Research Program NPRP award [NPRP-06-1208-2-492] from the Qatar National Research Fund (a member of The Qatar Foundation). The statements made herein are solely the responsibility of the author(s).

593

594

The authors are grateful to Prof. Chris Gold for discussion related to this work and his valuable suggestions.

\section{References}

Afyouni, I., Cyril, R. and Christophe, C., 2012. Spatial models for context-aware indoor navigation systems: A survey. Journal of Spatial Information Science, 1(4): 85--123.

Aurenhammer, F., 1991. Voronoi diagrams\&mdash;a survey of a fundamental geometric data structure. ACM Comput. Surv., 23(3): 345-405.

Axelsson, P., 2000. DEM Generation from Laser Scanner Data Using Adaptive TIN Models. In: D. Fritsch and M. Molenaar (Editors), XIXth ISPRS Congress. ISPRS, Amsterdam, The Netherlands, pp. 110-117.

Becker, T., Nagel, C. and Kolbe, T.H., 2009. A Multilayered space-event model for navigation in indoor spaces. 3D Geo-Information Sciences: 61-77.

Boguslawski, P., 2011. Modelling and Analysing 3D Building Interiors with the Dual Half-Edge Data Structure. PhD Thesis, University of Glamorgan, Pontypridd, Wales, UK, 134 pp.

Boguslawski, P. and Gold, C., 2010. Euler Operators and Navigation of Multi-shell Building Models. In: T. Neutens and P. Maeyer (Editors), Developments in 3D Geo-Information Sciences. Lecture Notes in Geoinformation and Cartography. Springer, pp. 1-16.

Boguslawski, P. and Gold, C., 2015. Buildings and terrain unified - multidimensional dual data structure for GIS. Geo-spatial Information Science, 18(4): 151-158.

Boguslawski, P., Gold, C.M. and Ledoux, H., 2011. Modelling and analysing 3D buildings with a primal/dual data structure. ISPRS Journal of Photogrammetry and Remote Sensing, 66(2): 188-197.

Brown, G., Nagel, C., Zlatanova, S. and Kolbe, T.H., 2013. Modelling 3D Topographic Space Against Indoor Navigation Requirements. In: J. Pouliot, S. Daniel, F. Hubert and A. Zamyadi (Editors), Progress and New Trends in 3D Geoinformation Sciences. Springer Berlin Heidelberg, Berlin, Heidelberg, pp. 1-22.

Chedid, R. and Najjar, N., 1996. Automatic finite-element mesh generation using artificial neural networks-Part I: Prediction of mesh density. IEEE Transactions on Magnetics, 32(5): 51735178.

DeCapua, C. and Bhaduri, B., 2007. Applications of Geospatial Technology in International Disasters and During Hurricane Katrina, Available at the Project Site of Capturing Hurricane Katrina Data For Analysis and Lessons-Learned Research (www.gri.msstate.edu/research/katrinalessons/Documents/GeoSp Tech Applications.pdf).

Du, Q., Faber, V. and Gunzburger, M., 1999. Centroidal Voronoi Tessellations: Applications and Algorithms. SIAM Review, 41(4): 637-676.

Du, Q. and Gunzburger, M., 2002. Grid generation and optimization based on centroidal Voronoi tessellations. Applied Mathematics and Computation, 133(2-3): 591-607.

Dyck, D.N., Lowther, D.A. and McFee, S., 1992. Determining an approximate finite element mesh density using neural network techniques. IEEE Transactions on Magnetics, 28(2): 1767-1770.

Goetz, M. and Zipf, A., 2011. Formal definition of a user-adaptive and length-optimal routing graph for complex indoor environments. Geo-spatial Information Science, 14(2): 119-128.

Green, P.J. and Sibson, R., 1978. Computing Dirichlet Tessellations in the Plane. The Computer Journal, 21(2): 168-173. 
Ho-Le, K., 1988. Finite element mesh generation methods: a review and classification. ComputerAided Design, 20(1): 27-38.

Krūminaitè, M. and Zlatanova, S., 2014. Indoor space subdivision for indoor navigation, Proceedings of the Sixth ACM SIGSPATIAL International Workshop on Indoor Spatial Awareness. ACM, Dallas/Fort Worth, Texas, pp. 25-31.

Kuligowski, E.D.I., 10-11 June 2004. p. 68-90., 2004. Review of 28 egress models. In: R.D. Peacock and E.D. Kuligowski (Editors), The workshop on building occupant movement during fire emergencies, 10-11 June 2004. National Institute of Standards and Technology, pp. 66-88.

Kwan, M.-P. and Lee, J., 2005. Emergency response after 9/11: the potential of real-time 3D GIS for quick emergency response in micro-spatial environments. Computers, Environment and Urban Systems, 29: 93-113.

Lamarche, F. and Donikian, S., 2004. Crowd of Virtual Humans: a New Approach for Real Time Navigation in Complex and Structured Environments. Computer Graphics Forum, 23(3): 509518.

Lee, J., 2004. A spatial access-oriented implementation of a 3-D GIS topological data model for urban entities. Geoinformatica, 8(3): 237-264.

Lee, J. and Kwan, M.P., 2005. A combinatorial data model for representing topological relations among 3D geographical features in micro-spatial environments. International Journal of Geographical Information Science, 19(10): 1039 - 1056.

Li, N., Becerik-Gerber, B., Krishnamachari, B. and Soibelman, L., 2014. A BIM centered indoor localization algorithm to support building fire emergency response operations. Automation in Construction, 42: 78-89.

Liu, L. and Zlatanova, S., 2011. A "door-to-door" path-finding approach for indoor navigation, Gi4DM 2011: Geolnformation for Disaster Management. International Society for Photogrammetry and Remote Sensing (ISPRS), Antalya, Turkey.

Liu, L. and Zlatanova, S., 2012. Towards a 3D network model for indoor navigation. In: Zlatanova, Ledoux, Fendel and Rumor (Editors), Urban and Regional Data Management. UDMS Annual 2011. CRCpress/Taylor and Francis Group, London, pp. 79-92.

Luo, F., Cao, G. and Li, X., 2014. An interactive approach for deriving geometric network models in 3D indoor environments, Proceedings of the Sixth ACM SIGSPATIAL International Workshop on Indoor Spatial Awareness. ACM, Dallas/Fort Worth, Texas, pp. 9-16.

Mostafavi, M.A., Gold, C. and Dakowicz, M., 2003. Delete and insert operations in Voronoi/Delaunay methods and applications. Computers \& Geosciences, 29(4): 523-530.

Nelson, H.E. and MacLennan, H.A., 1995. Emergency Movement. In: P.J. DiNenno, C.L. Beyler, R.L.P. Custer, W.D. Walton, J.M. Watts, D. Drysdale and J.R. Hall (Editors), SFPE handbook of fire protection engineering. Society of Fire Protection Engineers, Boston, MA, USA and National Fire Protection Association, Quincy, MA, USA, pp. 3/286-3/295.

OGC, 2014. OGC IndoorGML. Open Geospatial Consortium Inc.

Pauls, J., 1995. Movement of people. In: P.J. DiNenno, C.L. Beyler, R.L.P. Custer, W.D. Walton, J.M. Watts, D. Drysdale and J.R. Hall (Editors), SFPE handbook of fire protection engineering. Society of Fire Protection Engineers, Boston, MA, USA and National Fire Protection Association, Quincy, MA, USA, pp. 3/263-3/285.

Petersen, S.B. and Martins, P.A.F., 1997. FINITE ELEMENT REMESHING: A METAL FORMING APPROACH FOR QUADRILATERAL MESH GENERATION AND REFINEMENT. International Journal for Numerical Methods in Engineering, 40(8): 1449-1464.

Scharfenort, N., 2012. Urban Development and Social Change in Qatar: The Qatar National Vision 2030 and the 2022 FIFA World Cup. Journal of Arabian Studies, 2(2): 209-230.

Stoffel, E.P., Lorenz, B. and Ohlbach, H.J., 2007. Towards a Semantic Spatial Model for Pedestrian Indoor Navigation Advances in Conceptual Modeling - Foundations and Applications. Lecture Notes in Computer Science, pp. 328-337.

Stroud, I., 2006. Boundary Representation Modelling Techniques. Springer-Verlag, New York. 
Tashakkori, H., Rajabifard, A. and Kalantari, M., 2015. A new 3D indoor/outdoor spatial model for indoor emergency response facilitation. Building and Environment, 89: 170-182.

Vanclooster, A., De Maeyer, P., Fack, V. and Van de Weghe, N., 2014. Calculating Least Risk Paths in 3D Indoor Space. In: U. Isikdag (Editor), Innovations in 3D Geo-Information Sciences. Lecture Notes in Geoinformation and Cartography. Springer International Publishing, pp. 13-31.

Wallgrün, J., 2005. Autonomous Construction of Hierarchical Voronoi-Based Route Graph Representations. In: C. Freksa, M. Knauff, B. Krieg-Brückner, B. Nebel and T. Barkowsky (Editors), Spatial Cognition IV. Reasoning, Action, Interaction. Lecture Notes in Computer Science. Springer Berlin Heidelberg, pp. 413-433.

Wang, Z. and Zlatanova, S., 2013. Taxonomy of Navigation for First Responders. In: M.J. Krisp (Editor), Progress in Location-Based Services. Springer Berlin Heidelberg, Berlin, Heidelberg, pp. 297-315.

Wenjie, Y. and Schneider, M., 2010. Supporting Continuous Range Queries in Indoor Space, Eleventh International Conference on Mobile Data Management (MDM), pp. 209-214.

Yang, L. and Worboys, M., 2015. Generation of navigation graphs for indoor space. International Journal of Geographical Information Science, 29(10): 1737-1756.

Zhang, J. and Lin, X., 2013. Filtering airborne LiDAR data by embedding smoothness-constrained segmentation in progressive TIN densification. ISPRS Journal of Photogrammetry and Remote Sensing, 81: 44-59.

Zverovich, V., Mahdjoubi, L., Boguslawski, P., Fadli, F. and Barki, H., 2016. Emergency Response in Complex Buildings: Automated Selection of Safest and Balanced Routes. Computer-Aided Civil and Infrastructure Engineering. 